\title{
Concordância Entre as Medidas de Peso \\ e Estatura Mensuradas e Auto-Referidas \\ para o Diagnóstico do Estado Nutricional de Idosos Residentes no Sul do Brasil
}

\section{Agreement Between Self-Reported Weight and Height Measurements for the Diagnosis of the Nutritional Status of Older Residents in Southern Brazil}

\author{
Cassiano Ricardo Rech ${ }^{1}$ \\ Edio Luiz Petroski² \\ Othmar Böing ${ }^{3}$ \\ Rubens José Babel Júnior ${ }^{3}$ \\ Manoela Regina Soares
}

1. Ms. Professor Assistente, Departamento de Educação Física, Universidade Estadual de Ponta Grossa (UEPG), Ponta Grossa, Paraná, Brasil.

2. PhD. Professor Titular, Programa pós-graduação em Educação Física, Universidade Federal de Santa Catarina (UFSC), Florianópolis, Santa Catarina, Brasil.

3. Acadêmico do Curso de Educação Física da Universidade Federal de Santa Catarina (UFSC),

Florianópolis, Santa Catarina, Brasil.

\section{Endereço para correspondência: \\ R. Dezenove de Dezembro, 344 apt 102 - Centro - Ponta Grossa \\ - Paraná - Brasil - CEP 84010-390 \\ Fone: (42- 32255464 ou 99693052) \\ Email: crrech@hotmail.com crrech@uepg.br}

Submetido em 18/10/2006 Versão final recebida em 06/07/2007 Aceito em 01/11/2007

\begin{abstract}
RESUMO
Este estudo teve como objetivos verificar a concordância entre as medidas mensuradas e auto-referidas de peso, estatura e índice de massa corporal (IMC) e analisar a influência do estado nutricional e das variáveis sociodemográficas na diferença da medida auto-referida em idosos. Foram avaliados 368 idosos (284 mulheres e 84 homens), entre 60 e 85 anos de idade. As medidas mensuradas e auto-referidas de peso e estatura foram coletadas, sendo que as informações auto-referidas e sociodemograficas foram obtidas por meio de um questionário auto-aplicado. Utilizou-se estatística descritiva e o teste t student para comparar a médias, análise de variância (ANOVA) para comparar as diferenças em relação ao estado nutricional e o teste de Kruskal-Wallis foi utilizado para comparar as diferenças em relação aos grupos de idade, nível educacional e nível socioeconômico. Utilizou-se o programa SPSS versão 11.0 com um nível de significância de $p<0,05$. Os homens tenderam a superestimar os valores de peso auto-referido. Nas mulheres, foram observadas diferenças significativas para todas as variáveis analisadas, sendo que a estatura auto-referida foi superestimada, enquanto que o peso e o IMC foram subestimados sistematicamente. As prevalências de sobrepeso para homens e mulheres diferiram significativamente, sendo influenciada nas mulheres pela idade e pelo nível educacional, já homens, apenas pela idade. Uma diferença inferior a $2 \mathrm{~kg} / \mathrm{m}^{2}$ no IMC auto-referido foi observado em 89,3\% dos homens e 80,9\% das mulheres. Com isso, concluiu-se que o IMC auto-referido apresentou elevada concordância para avaliação nutricional em homens idosos; contudo, entre as mulheres idosas a utilização das medidas auto-referidas subestimaram a prevalência de sobrepeso e obesidade.
\end{abstract}

Palavras-chave: índice de massa corporal, medidas auto-referidas, idosos.

\begin{abstract}
The objectives of this study were to verify the agreement between the self-reported weight, height and body mass index (BMI) measurements of older adults and to analyze the influence of nutritional status and socio-demographic variables on the error in those measurements. A total of 368 older subjects (284 women and 84 men), aged 60-85 years were evaluated. Weight and height were measured and the selfreported measurements were collected together with socio-demographic data obtained by a self-administered questionnaire. Data were analyzed using descriptive statistics, the Student's t test to compare means, analysis of variance (ANOVA) to compare the relationship between differences and nutritional status and the Kruskal-Wallis test to compare the relationship between differences and in age, educational background and socio-economic status. Analyses were performed using SPSS version 11.0 and the level of significance was set at $p<0.05$. Men tended to overestimate their own weight. Among women, significant differences were observed for all studied variables, with height being overestimated and weight and BMI being systematically underestimated. Prevalence rates of overweight among men and women differed significantly, being influenced by age and educational background among women, but only by age among men. An error of less than $2 \mathrm{~kg} / \mathrm{m}^{2}$ in self-reported BMI was observed in $89.3 \%$ of the men and $80.9 \%$ of the women. Therefore, it was concluded that self-referred BMI presented high agreement for the nutritional assessment of male older adults, whereas among females the use of self-reported measurements underestimated the prevalence of overweight and obesity.
\end{abstract}

Keywords: body mass index, self-reported measurements, older adults. 


\section{INTRODUÇÃO}

As medidas de peso e estatura, além de serem utilizadas para analisar fatores de crescimento, desenvolvimento e maturação, têm sido, cada vez mais, usadas para avaliar o estado nutricional (desnutrição, sobrepeso ou obesidade) na população em geral(1-3).

Nesse sentido, a antropometria, que é um método não invasivo e de fácil aplicação, têm sido de grande valia no diagnóstico do estado nutricional. Nos últimos anos, uma nova alternativa vem sendo utilizada no levantamento de informações antropométricas (peso e estatura), é o que a literatura chama de peso e estatura auto-referidos ou informados, ou seja, uma autopercepção dessas medidas ${ }^{(4)}$ que tem tornado possível estudos epidemiológicos em grandes populações, favorecendo a economia de recurso e a simplificação do trabalho de campo.

Porém, quando se utiliza uma medida auto-referida, as diferenças a ela associada tendem a ser maior do que quando a mensuração é realizada. Deste modo, alguns estudos procuraram validar as medidas de peso e estatura auto-referidas em várias populações ${ }^{(5-10)}$, comprovando que este procedimento fornece dados válidos e confiáveis, até mesmo em indivíduos obesos ou grupos com uma maior vulnerabilidade de acesso às informações de peso e estatura, como por exemplo, pessoas com baixa escolaridade ${ }^{(1)}$. Observa-se que algumas variáveis estão relacionadas com erros nas medidas auto-referidas, tais como: idade, sexo, renda, escolaridade entre outras que, quando associadas, explicam parte destas diferenças ${ }^{(4,12,13)}$.

Investigações apontam que, entre os homens, ocorre uma superestimativa da estatura ${ }^{(14)}$, e entre as mulheres, uma subestimativa do peso e do índice de massa corporal(5). No Brasil, ainda são poucos os estudos que utilizam medidas auto-referidas. Fonseca et al. ${ }^{(11)}$ apresentaram uma alta concordância entre os valores mensurados e auto-referidos de peso e estatura, em indivíduos adultos da cidade do Rio de Janeiro. Schmidt et al. ${ }^{(15)}$ analisaram a população urbana da cidade de Porto Alegre e encontraram associação significativa entre os valores mensurados e auto-referidos. Porém, os homens tenderam superestimar o valor do peso corporal e as mulheres subestimá-lo.

Em ambos os estudos, a idade esteve associada com os erros nas medidas auto-referidas, evidenciando-se, em pessoas acima de 50 anos, erros significativos entre os valores mensurados e auto-referidos $(4,13,16)$. Um dos principais pontos considerados com relação à idade refere-se ao processo de envelhecimento que é acompanhado por mudanças na composição corporal e na redistribuição dos tecidos, que é resultante do decréscimo da massa magra, da água corporal, aumento do tecido adiposo e diminuição da estatura ${ }^{(17)}$. Com isso, em idosos, as medidas auto-referidas têm apresentado uma superestimativa da estatura e subestimativa do peso e do índice de massa corporal, diminuindo a estimativa da prevalência de sobrepeso e obesidade ${ }^{(4,16)}$.

Assim, este estudo teve como objetivos: a) analisar a concordância entre as medidas mensuradas e auto-referidas de peso, estatura e índice de massa corporal em idosos; b) analisar a influência das variáveis sociodemográficas (sexo, idade, renda e escolaridade) na diferença das medidas auto-referidas; c) comparar as prevalências de sobrepeso e obesidade obtidas através das medidas auto-referidas e mensuradas.

\section{MÉTODOS}

Com o objetivo de analisar as diferenças associadas às medidas auto-referidas de peso e estatura em idosos, comparando com os valores mensurados, desenvolveu-se um estudo transversal de cunho descritivo exploratório ${ }^{(19)}$. Participaram do estudo idosos de ambos os sexos, com idade acima de 60 anos, participantes dos Grupos de Terceira Idade do Município de Florianópolis-SC. Os grupos de terceira idade referem-se a grupos organizados que têm como objetivo for- necer atividades físicas, culturais e de lazer para os idosos. De acordo com o levantamento realizado em 2005, no momento da seleção da amostra, estavam cadastrados nos grupos de terceira idade cerca de 3182 idosos.

Adotou-se para o cálculo amostral mínimo um erro de 5\%, considerando uma perda de $10 \%$ dos valores como margem de segurança. Foi observada uma perda de $6 \%$ do total de dados coletados (23 indivíduos), em função do não preenchimento dos valores de peso e estatura referidos ou de algumas das variáveis sociodemográficas. Deste modo, a amostra final foi composta por 368 idosos (284 mulheres e 84 homens), com idade entre 60 e 85 anos.

Importante salientar que não foram incluídos na amostra indivíduos institucionalizados. Além disso, todos os idosos foram voluntários e assinaram o Termo de Consentimento Livre e Esclarecido, obedecendo às normas para realização de pesquisa em seres humanos, resolução 196/96, do Conselho Nacional de Saúde.

As variáveis sociodemográficas (sexo, idade, renda e escolaridade) e as informações de peso e estatura auto-referidas foram obtidas por meio de um questionário aplicado, nos grupos de Terceira Idade, de forma dirigida, em grupos de até cinco pessoas ou individualmente para que fossem diminuídos os erros provenientes deste tipo de instrumento, levando em conta as características da amostra (idosos).

Posteriormente, os sujeitos tiveram seu peso e estatura mensurados. Para o peso foi usada uma balança digital, marca Plenna, com $150 \mathrm{~kg}$ de capacidade e resolução de 100g, com o indivíduo vestindo o mínimo de roupa e descalço. A estatura foi mensurada por meio de uma fita métrica fixada na parede verticalmente. Para ambas as medidas, foram adotados os procedimentos descritos por Alvarez e Pavan ${ }^{(20)}$.

O índice de massa corporal (IMC), que representa a razão do peso em quilogramas pelo quadrado do valor da estatura em metros, foi calculado com a utilização das medidas mensuradas e auto-referidas, sendo usado para análise das prevalências de sobrepeso e obesidade e foi classificado segundo critérios da Organização Mundial da Saúde (OMS)(21): IMC < 18,5 kg/m² (baixo peso); IMC $\geq 18,5 \mathrm{~kg} / \mathrm{m}^{2}$ até 24,9 $\mathrm{kg} / \mathrm{m}^{2}$ (eutrófico); IMC $\geq 25 \mathrm{~kg} / \mathrm{m}^{2}$ até $29,9 \mathrm{~kg} / \mathrm{m}^{2}$ (sobrepeso) e IMC $\geq 30 \mathrm{~kg} / \mathrm{m}^{2}$ (obeso).

A idade foi utilizada como variável contínua na maioria das análises, quando categorizada, foi ordenada em cinco faixas etárias: 60-64,9 anos; 65-69,9 anos; 70-74,9 anos; 75-79,9 anos e 80-85 anos. Para análise da renda, foi utilizada a renda domiciliar percapita em salários mínimos (o salário vigente na época da pesquisa era de $\mathrm{R} \$ 300,00$ reais), sendo utilizada a seguinte categorização: menos de dois salários, entre dois e quatro salários, entre quatro e seis salários e acima de seis salários. Com relação à escolaridade, foram adotadas quatro categorias: primário incompleto, primário completo/ginásio incompleto, ginásio completo/superior incompleto e superior completo.

Para análise da diferença entre a média dos valores auto-referidos e mensurados, utilizou-se o teste " $t$ " pareado, o coeficiente de correlação intraclasse ${ }^{(22)}$, e a análise gráfica de Bland e Altman ${ }^{(23)}$. Comparou-se a diferença entre as médias dos grupos em função do estado nutricional, por meio da análise de variância para um fator (ANOVA), quando a análise apresentou diferença entre os grupos, foi aplicado o teste Post-Hoc de Tukey, a fim de identificar os grupos que apresentaram a diferença estatística. Utilizou-se o teste Kruskal-Wallis para analisar as diferenças entres as diferenças médias em função das variáveis categóricas (sociodemográficas e idade).

Além disso, foram calculadas as prevalências de sobrepeso e obesidade, comparando os valores mensurados e auto-referidos através da análise do teste crítico das razões (Z) e para identificar a concordância dos valores mensurados e referidos, foi analisado o cálculo do índice 
de sensibilidade e especificidade para o diagnóstico de sobrepeso e obesidade das medidas auto-referidas em relação às medidas mensuradas. Para as análises, foi utilizado o pacote estatístico SPSS 11.0, adotando-se um nível de significância de 5\%.

\section{RESULTADOS}

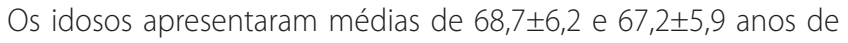
idade, para o sexo masculino e feminino, respectivamente. Quanto às características da amostra, foram assim distribuídas: $77,1 \%$ dos avaliados são do sexo feminino, 85,6\% encontram-se entre 60 e 75 anos. Em relação ao estado civil, $62,1 \%$ são casados ou vivem com parceiro e apenas 5,3\% são solteiros, entre os homens, 86,9\% são casados. A renda apresentou uma distribuição homogênea entre as categorias, sendo que 18,7\% declararam receber menos de dois salários mínimos, 27,6\% entre dois e quatro salários, 28,1\% entre quatro e seis salários e 28,1\% relataram uma renda superior a seis salários mínimos. A maior parte dos idosos $(50,4 \%)$ relatou um tempo de estudo que corresponde ao primário completo e 16\% apresentaram curso superior.

Com relação ao estado nutricional, de acordo com os valores analisados e a classificação da OMS(21), observou-se que $28,8 \%$ são eutróficos, $51,1 \%$ apresentam sobrepeso e 18,7\% obesidade.

Quando analisadas em termos gerais (tabela 1), as variáveis autoreferidas apresentaram uma subestimativa do peso e, conseqüentemente, do IMC, já a estatura foi superestimada e média em 0,87cm. Contudo, os valores do coeficiente de correlação intraclasse foram altos para todas as variáveis. Quando analisado em relação ao sexo, notou-se que os erros foram distribuídos de forma diferenciada.

Nos homens, houve uma superestimativa de todas as variáveis, no entanto, somente o peso apresentou uma diferença significativa com um erro médio de $0,71 \mathrm{~kg}$. Para as mulheres, as diferenças entre os valores auto-referidos e mensurados diferiram significativamente para todas as variáveis (peso, estatura e IMC) $(p<0,01)$, tendo a estatura apresentado uma diferença média de $1,01 \mathrm{~cm}$. A correlação intraclasse foi significativa em ambos os sexos analisados $(p<0,01)$.

Com relação às diferenças em função do estado nutricional dos indivíduos (tabela 2), pode-se notar que os homens eutróficos apresentaram uma superestimativa das medidas de IMC auto-referido, enquanto que as mulheres apresentaram uma subestimativa dos va- lores de IMC, em média de 0,37kg/m². Em relação ao grupo de obesos, notou-se que mulheres obesas tendem a subestimar o IMC em 0,50kg/m², diferentemente dos homens obesos que apresentam uma superestimativa do IMC. Contudo, foi à categoria de sobrepeso em mulheres que apresentou a maior variação do IMC auto-referido.

A análise da diferença média entre os valores mensurados e auto-referidos para o IMC em relação às variáveis sociodemográficas (tabela 3) demonstra que entre os homens, as diferenças estão estatisticamente associadas somente com a idade (80-85 anos). Para as outras variáveis, não foram encontrados valores significativos. Já entre as mulheres, a idade e a baixa escolaridade foram às variáveis que apresentaram as maiores diferenças associados ao IMC. Sendo que, mulheres nas

Tabela 2. Valores de diferença média, coeficiente de correlação intraclasse (CCI) e intervalo de confiança (IC) de IMC, em relação ao estado nutricional de idosos participantes de Grupos de Terceira Idade de Florianópolis - SC.

\begin{tabular}{c|l|c|c|c|c}
\hline População & $\begin{array}{c}\text { Estado } \\
\text { nutricional }\end{array}$ & $\mathbf{n}$ & $\begin{array}{c}\text { diferença } \\
\text { média } \mp\end{array}$ & CCI & IC (95\%) \\
\hline Todos & & & & & \\
\hline & Eutrófico* & 106 & $-0,14$ & 0,98 & $0,97-0,98$ \\
\hline & Sobrepeso & 188 & $0,87^{* *}$ & 0,94 & $0,93-0,95$ \\
\hline & Obeso & 74 & $-0,34$ & 0,94 & $0,92-0,95$ \\
\hline Homens & & & & & \\
\hline & Eutrófico* & 20 & $0,64^{* *}$ & 0,98 & $0,94-0,97$ \\
\hline & Sobrepeso & 54 & 0,39 & 0,95 & $0,92-0,96$ \\
\hline Mulheres & Obeso & 10 & 0,17 & 0,90 & $0,85-0,94$ \\
\hline & Eutrófico & 86 & $-0,37$ & 0,98 & $0,97-0,98$ \\
\hline & Sobrepeso & 134 & $1,01^{* *}$ & 0,89 & $0,86-0,91$ \\
\hline & Obeso & 64 & $-0,50^{* *}$ & 0,94 & $0,93-0,95$ \\
\hline
\end{tabular}

* foram incluídos dois indivíduos com baixo peso.

** difere estatisticamente $p<0,05$ análise da Anova para um fator (Post Hoc de Tukey).

$\mp$ diferença média = média da diferença entre os valores auto-referidos e mensurados.

Tabela 1. Valores de média, desvio padrão, diferença média, coeficiente de correlação intraclasse (CCI) e intervalo de confiança (IC) para os valores de peso, estatura e IMC mensurados e auto-referidos, em idosos participantes de Grupos de Terceira Idade de Florianópolis - SC.

\begin{tabular}{|c|c|c|c|c|c|c|}
\hline População & Variável & auto-referido & mensurado & diferença média $\mp$ & $\mathrm{CCl}$ & IC (95\%) \\
\hline \multirow{3}{*}{ Todos } & Peso (kg) & $67,7 \pm 11,2$ & $67,9 \pm 11,2$ & $-0,19$ & 0,98 & $0,97-0,98$ \\
\hline & Estatura $(\mathrm{cm})$ & $159,1 \pm 8,6$ & $158,2 \pm 8,1$ & $0,87^{* * *}$ & 0,94 & $0,93-0,95$ \\
\hline & IMC $\left(\mathrm{kg} / \mathrm{m}^{2}\right)$ & $26,7 \pm 3,8$ & $27,08 \pm 3,7$ & $-0,62^{* * *}$ & 0,94 & $0,92-0,95$ \\
\hline \multirow{3}{*}{ Homens } & Peso (kg) & $76,1 \pm 10,6$ & $75,4 \pm 10,3$ & $0,71^{* *}$ & 0,98 & $0,94-0,97$ \\
\hline & Estatura $(\mathrm{cm})$ & $168,4 \pm 8,1$ & $168,1 \pm 7,3$ & 0,39 & 0,95 & $0,92-0,96$ \\
\hline & $\mathrm{IMC}\left(\mathrm{kg} / \mathrm{m}^{2}\right)$ & $26,82 \pm 2,9$ & $26,64 \pm 2,8$ & 0,17 & 0,90 & $0,85-0,94$ \\
\hline \multirow{3}{*}{ Mulheres } & Peso (kg) & $65,3 \pm 10,1$ & $65,7 \pm 10,4$ & $-0,37^{* * *}$ & 0,98 & $0,97-0,98$ \\
\hline & Estatura $(\mathrm{cm})$ & $156,3 \pm 6,6$ & $155,3 \pm 5,8$ & $1,01^{* * *}$ & 0,89 & $0,86-0,91$ \\
\hline & IMC $\left(\mathrm{kg} / \mathrm{m}^{2}\right)$ & $26,7 \pm 4,03$ & $27,2 \pm 3,9$ & $-0,50^{* * *}$ & 0,94 & $0,93-0,95$ \\
\hline
\end{tabular}

** difere estatisticamente $\mathrm{p}<0,05$ análise do teste t pareado.

*** difere estatisticamente $p<0,01$ análise do teste $t$ pareado.

₹ diferença média = média da diferença entre os valores auto-referidos e mensurados. 
Tabela 3. Número de sujeitos e valores de diferença média para a comparação entre médias do IMC, em relação as variáveis sociodemográficas de idosos participantes de Grupos de Terceira Idade de Florianópolis - SC.

\begin{tabular}{|c|c|c|c|c|}
\hline \multirow[b]{2}{*}{ Variáveis } & \multicolumn{2}{|c|}{ Masculino } & \multicolumn{2}{|c|}{ Feminino } \\
\hline & $n$ & $\begin{array}{c}\text { diferença } \\
\text { média }^{\mp}\end{array}$ & $\mathrm{n}$ & $\begin{array}{c}\text { diferença } \\
\text { média }^{\mp}\end{array}$ \\
\hline \multicolumn{5}{|l|}{ Idade * } \\
\hline $60-65$ & 27 & $0,21^{\mathrm{a}}$ & 108 & $-0,47^{a}$ \\
\hline $65-70$ & 14 & $0,35^{a}$ & 78 & $-0,31^{a}$ \\
\hline $70-75$ & 27 & $-0,23 a$ & 61 & $-0,61^{b}$ \\
\hline $75-80$ & 10 & $0,31^{\mathrm{a}}$ & 27 & $-0,77^{b}$ \\
\hline $80-85$ & 06 & $1,17^{b}$ & 10 & $-0,64^{b}$ \\
\hline \multicolumn{5}{|l|}{ Escolaridade * } \\
\hline Ginasial incompleto & 19 & 0,01 & 94 & $-0,72^{b}$ \\
\hline Colegial incompleto & 21 & 0,64 & 67 & $-0,21^{a}$ \\
\hline Colegial completo & 27 & 0,17 & 81 & $-0,54^{a}$ \\
\hline Superior & 17 & $-0,25$ & 42 & $-0,31^{a}$ \\
\hline \multicolumn{5}{|l|}{ Renda } \\
\hline$<2$ salários & 12 & $-0,38$ & 64 & 0,51 \\
\hline 2 e 4 salários & 22 & 0,37 & 77 & 0,64 \\
\hline 4 e 6 salários & 19 & 0,71 & 82 & 0,28 \\
\hline > 6 salários & 31 & 0,09 & 61 & 0,57 \\
\hline
\end{tabular}

* difere estatisticamente $p<0,05$ análise do teste de comparação de Kruskal-Wallis, letras diferentes representam que os grupos diferem significativamente.

${ }^{\mp}$ diferença média = média da diferença entre os valores auto-referidos e mensurados.

categorias entre 70-85 anos apresentaram uma subestimativa média entre 0,61 e 0,77 kg/m² no IMC auto-referido.

Após a determinação dos valores de IMC mensurado e auto-referido, as taxas de sensibilidade e especificidade foram calculadas a fim de identificar a concordância entre os diagnósticos de sobrepeso e obesidade dos valores mensurados e auto-referidos. Pode-se observar, na tabela 4, que os valores de sensibilidade e especificidade foram altos, tanto para sobrepeso quanto para obesidade.

As prevalências de sobrepeso e obesidade, no sexo feminino, foram de $47,2 \%$ e 22,5\% respectivamente, para os valores mensurados e 41,5\% e $20,8 \%$ para os valores auto-referidos. Em relação ao sexo masculino, na categoria de sobrepeso e obesidade, a prevalência é de 64,3\% e $11,9 \%$ para os valores mensurados e $57,1 \%$ e $11,9 \%$ para os valores auto-referidos, respectivamente.

Na análise do teste crítico das proporções, que compara duas prevalências e analisa a significância das diferenças observadas, tanto para o sexo masculino quanto para o sexo feminino, as prevalências

Tabela 4. Sensibilidade e Especificidade do IMC auto-referido no diagnóstico de sobrepeso e obesidade em idosos participantes de Grupos de Terceira Idade de Florianópolis - SC

\begin{tabular}{l|c|c|c|c}
\hline \multirow{2}{*}{ Variável } & \multicolumn{2}{|c|}{ Masculino } & \multicolumn{2}{c}{ Feminino } \\
\cline { 2 - 5 } & Sensibilidade & Especificidade & Sensibilidade & Especificidade \\
\hline Sobrepeso & $93 \%$ & $65 \%$ & $83 \%$ & $92 \%$ \\
\hline Obesidade & $70 \%$ & $92 \%$ & $83 \%$ & $95 \%$ \\
\hline
\end{tabular}

Para a classificação do estado nutricional adotou-se os seguintes pontos de corte: sobrepeso $\geq 25 \mathrm{~kg} / \mathrm{m}^{2}$ e obesidade $\geq 30 \mathrm{~kg} / \mathrm{m}^{2}$. de sobrepeso, calculadas a partir dos valores auto-referidos, foram estatisticamente diferentes $(Z=2,35 ; p<0,05)$.

Ainda relacionado à dispersão das diferenças nas medidas auto-referidas, a figura 1 apresenta os valores da diferença média e o intervalo de confiança (95\%) para estatura, peso e IMC (auto-referido menos mensurado), para homens e mulheres, nota-se que para a estatura, homens (gráfico A) e mulheres (gráfico B) apresentaram 10,7\% e 28,1\% respectivamente, da amostra com valores de diferença entre $\pm 3 \mathrm{~cm}$. No entanto, 40,4\% das mulheres apresentam diferenças iguais ou superiores a $2 \mathrm{~cm}$ em relação às medidas mensuradas.

Quanto à análise dos valores de peso corporal auto-referido, 16,6\% dos homens (gráfico C) e 13,38\% das mulheres (gráfico D) apresentaram uma diferença no peso igual ou superior a $2 \mathrm{~kg}$, em relação às medidas mensuradas. Com relação ao IMC, 89,29\% dos homens (gráfico E) avaliados apresentaram uma diferença inferior à $2 \mathrm{~kg} / \mathrm{m}^{2}$. Já entre as mulheres (gráfico F), 80,9\% relataram uma diferença menor do que $2 \mathrm{~kg} / \mathrm{m}^{2}$ e $8,84 \%$ das mulheres apresentaram erro igual ou superior a $3 \mathrm{~kg} / \mathrm{m}^{2}$.

\section{DISCUSSÃO}

A utilização das medidas auto-referidas tem sido proposta como uma alternativa viável para o diagnóstico do estado nutricional, em estudos epidemiológicos em adultos, em diferentes populações ${ }^{(11,14,15,24)}$. Porém, em idosos, essa validade tem sido vista com cautela, devido aos erros associados ao envelhecimento ${ }^{(4,18)}$.

Uma das questões levantadas pela constância nas diferenças das medidas auto-referidas em idosos é em relação à diminuição da estatura em cerca de $1-2 \mathrm{~cm}$ por década ${ }^{(17)}$. Contudo, as variações causadas no peso parecem ser mais significativas para o cálculo do IMC. Assim, uma das preocupações em analisar a validade das medidas auto-referidas está em estabelecer a magnitude e o sentido das alterações causadas no cálculo da prevalência de baixo peso, sobrepeso e obesidade da população. Essa linha de raciocínio foi desenvolvida no estudo de Silveira et al. ${ }^{(16)}$, conduzido com a população adulta de Pelotas-RS, onde foram observadas mudanças significativas nas prevalências de sobrepeso e obesidade em mulheres, utilizando as medidas auto-referidas.

No presente estudo, os homens superestimaram os valores de peso, estatura e IMC, no entanto, somente o peso demonstrou diferença significativa. Esses achados diferem dos encontrados em estudos com populações de franceses ${ }^{(8)}$, adultos mexicanos ${ }^{(6)}$, adultos e idosos escoceses ${ }^{(9)}$, que observaram uma subestimativa do peso corporal. Já os estudos de Maranhão-Neto(14) e Shmitd et al. ${ }^{(15)}$ observaram a mesma tendência, em homens, de superestimação do peso e do IMC. No presente estudo, nos homens, a estatura auto-referida e mensurada não apresentou diferença significativa $(p<0,05)$, contudo a mesma foi superestimada, concordando com os relatos na literatura $(4,11,15,16,18)$.

Em relação ao sexo feminino, os resultados de subestimação do peso e do IMC, como a superestimativa da estatura, estão de acordo com o que apresenta a literatura ${ }^{(4,7,8)}$. Esse fato é mais evidenciado em mulheres, possivelmente pela busca do padrão estético, que faz a autopercepção de peso ideal diminuir os valores reais. Já na informação da estatura em idosos, os mesmos desconsideram as alterações com a idade, assim tendem a superestimar essa medida.

Pode-se observar também que idosos mais pesados, tendem a subestimar significativamente os valores de IMC auto-referido, principalmente em mulheres ${ }^{(15)}$. No presente estudo, observou-se que homens obesos tendem a superestimar o peso, enquanto que mulheres obesas e eutróficas subestimam o peso corporal e, consequentemente, a estimativa do IMC auto-referido. Isso deve ser considerado quando as medidas auto-referidas são utilizadas em grupos específicos, como por 


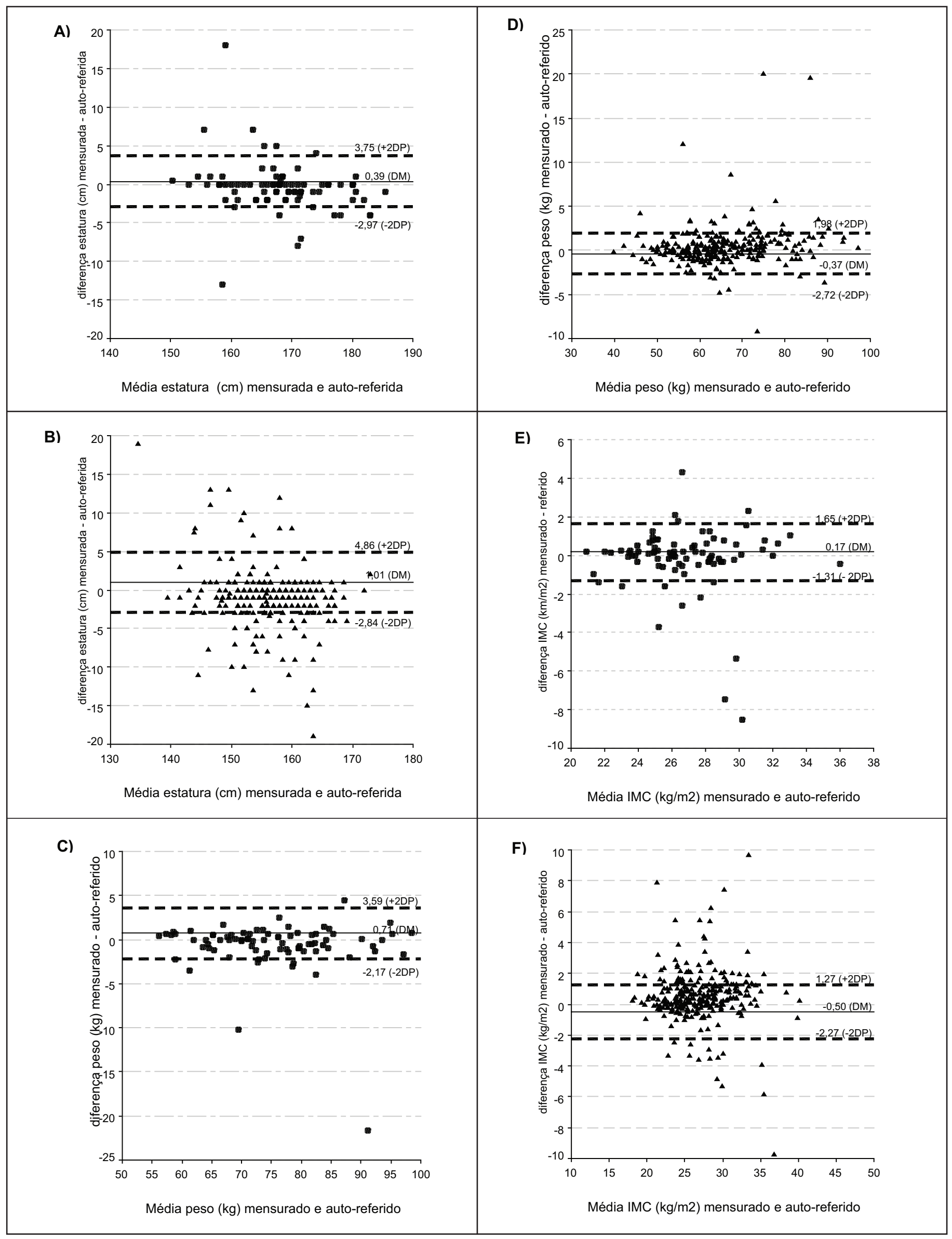

Figura 1. Análise gráfica da diferença média (EM) e Intervalo de confiança (95\%) dos valores auto-referidos menos valores mensurados, em relação à média dos valores autoreferidos e mensurados em idosos, participantes de Grupos de Terceira Idade de Florianópolis - SC. Os gráficos A, C e E representam a análise de estatura, peso e IMC para o sexo masculino, respectivamente. Os gráficos B, D e F representam a análise de estatura, peso e IMC para o sexo feminino, respectivamente. EM= erro médio. 2DP= dois desvios padrões. 
exemplo, em mulheres obesas, pois nestes casos o IMC auto-referido, difere estatisticamente das medidas mensuradas.

Além do estado nutricional, a idade se mostrou como uma variável associada às diferenças nas medidas auto-referidas (tabela 4). Para mulheres entre 70-85 anos e em homens entre 80-85 anos, essas diferenças foram significativas $(p<0,05)$, quando comparadas com idosos com menos idade. Esses resultados reforçam os achados de Kuczmarski et al. ${ }^{(4)}$, Ávila-Funes et al. ${ }^{(6)}$ e Niedhmmer et al. ${ }^{(8)}$ que relatam maiores diferenças em indivíduos com mais idade, principalmente quando se trata de mulheres com baixo nível de escolaridade. A renda não demonstrou ser uma variável que causasse erros significativos no IMC auto-referido, tanto em homens quanto em mulheres.

Quanto à análise do índice de sensibilidade, que estabelece o poder do teste em identificar um caso de diagnóstico "verdadeiro", no presente estudo, foi utilizado o IMC mensurado para diagnosticar, caso "verdadeiro" de sobrepeso ou obeso(22). Esta análise demonstrou que, na amostra de 284 mulheres avaliadas, o teste (IMC auto-referido) não conseguiu diagnosticar 48 casos de sobrepeso, que foram classificados como eutróficos ou obesos. A especificidade, que se refere ao poder do teste em identificar sujeitos que não apresentam "a doença", ou seja, não apresentam sobrepeso ou obesidade ${ }^{(22)}$. Neste estudo, os homens apresentam uma especificidade de $92 \%$, assim, somente $8 \%$ dos casos seriam classificados como obesos, quando na verdade apenas estariam com sobrepeso.

Os valores de sensibilidade e especificidade encontrados foram considerados altos para a concordância entre as medidas, indo ao encontro da literatura ${ }^{(5)}$. Quanto à variação dos erros, Schmidt et al.(15) relataram que $62 \%$ da amostra de adultos, da cidade de Porto Alegre$\mathrm{RS}$, apresentou um erro menor do que $2 \mathrm{~kg}$ para o peso. No presente estudo, um erro superior a $2 \mathrm{~kg}$ no peso foi observado em 16,6\% dos homens e 13,38\% das mulheres. Em suma, 80,9\% das mulheres e $89,29 \%$ dos homens, relataram um IMC com erro inferior a $2 \mathrm{~kg} / \mathrm{m}^{2} \mathrm{O}$ que segue os achados para adultos ${ }^{(11,16,24)}$.

Neste estudo, as prevalências de sobrepeso e obesidade foram subestimadas quando adotadas as medidas auto-referidas. As prevalências encontradas no grupo estudado, são diferentes das relatadas por Tavares e Anjos ${ }^{(2)}$ para indivíduos urbanos da região Sul do Brasil,

\section{REFERÊNCIAS BIBLIOGRÁFICAS}

1. Anjos LA. Índice de massa corporal (massa corporal.estatura-2) como indicador do estado nutricional de adultos: revisão de literatura. Rev Saúde Públ 1992; 26: 203-15.

2. Tavares EL, Anjos LA. Perfil antropométrico da população idosa brasileira. Resultados da pesquisa Nacional sobre Saúde e Nutrição. Cad Saúde Pública 1999; 15: 759-68.

3. Acuña $K$, Cruz T. Avaliação do estado nutricional de adultos e idosos e situação nutricional da população brasileira. Arq Bras Endocrinol Metab 2004; 48: 345-61.

4. Kuczmarki MF, Kuczmarski RJ, Najar M. Effects of age on validity of self-reported height, weight and body mass index: findings from the Third National Health and Nutrition Examination Survey, 1988-1994. J Am Diet Assoc 2001; 101:28-34.

5. Wada K, Tamakoshi K, Tsunekawa T, Otsuka R, Zhang H, Murata C, Nagasawa N, et al. Validity of self-reported height and weight in a Japanese workplace population. Int J Obes Relat Metab Disord 2005; 29: 1093-9.

6. Avila-Funes JA, Gutierrez-Robledo LM, Rosales PDL. Validity of height and weight self-reported in Mexican adults: results from the national health and ageing study. J Nutr Health Againg 2004; 8: 355-61.

7. Villanueva EV. The validity of self-reported weight in US adults: a population based cross-section study. BMC Public Health 2001; 1: 1-11.

8. Niedhammer I, Bugel I, Bonenfant S, Goldberg M, Leclerc A. Validity of self-reported weight and height in the Frech GAZEL cohort. Int J Obes Relat Metab Disord 2000; 24: 1111-8.

9. Bolton-Smith C, Woodward M, Tunstall-Pedoe H, Morrison C. Accuracy of the estimated prevalence of obesity from self reported height and weight in an adult Scottish population. J Epidemiol Community Health 2000; 54: 143-8.

10. Spencer EA, Appleby PN, Davey GK, Key TJ. Validity of self-reported height and weight in 4808 EPIC-Ox-ford participants. Public Health Nutr 2002; 5: 561-5.

11. Fonseca MJM, Faerstein E, Chor D, Lopes CS. Validade de peso e estatura informados e índice de massa corporal: um estudo pró-saúde. Rev Saúde Pública 2004; 38: 392-8.

12. Nawaz H, Chan W, Abdulrahman M, Larson D, Katz DL. Self-reported weight and height: implications for obesity research. Am J Prev Med 2001;20: 294-301. que apresentaram uma prevalência de 33\% de sobrepeso e 11,3\% para obesidade em homens, e 36\% de sobrepeso e 25,2\% para obesidade, em mulheres. Os valores deste estudo demonstraram um sobrepeso de $64,3 \%$ e 11,9\% de obesidade para homens e 41,5\% de sobrepeso e $20,8 \%$ de obesidade para mulheres.

Apesar das categorias (sobrepeso e obesidade) em ambos os sexos apresentarem uma subestimativa que variou de 7,2\% para o sobrepeso em homens e 1,7\% para a obesidade em mulheres, essa diferença foi significativa apenas para cálculos de sobrepeso quando analisado o teste crítico das razões $(p<0,05)$. Exceção se fez à classificação de homens classificados como obesos, que apresentaram o mesmo percentual, assim, possivelmente alguns casos de sobrepeso estão sendo classificados como eutróficos quando utilizadas as medidas auto-referidas.

Este estudo apresenta como pontos limitantes o fato de que os idosos participantes do estudo fazem parte de Grupos de Terceira Idade, que passam por avaliações físicas periódicas de peso e estatura. Assim, as menores diferenças provenientes das variáveis demográficas, podem ter sido compensados ou equiparados. Com isso, os resultados deste estudo aplicam-se a idosos não institucionalizados e participantes de grupos de terceira idade.

De acordo com os aspectos analisados em relação à utilização das medidas referidas em idosos, pode-se concluir que entre os homens há uma superestimativa do peso. As mulheres tendem a superestimar a estatura e subestimar o peso e o índice de massa corporal. As variáveis demográficas de idade e escolaridade se mostraram associadas aos erros das medidas auto-referidas em idosas, já para os homens, somente a idade esteve associada ao erro das medidas auto-referidas.

As prevalências de sobrepeso e obesidade foram subestimadas em mulheres idosas, enquanto que em homens somente a categoria de sobrepeso apresentou uma subestimativa quando utilizadas as medidas auto-referidas. Sugerem-se novos estudos que utilizem outras populações específicas de idosos para que os resultados apresentados no presente estudo possam ser confirmados.

Todos os autores declararam não haver qualquer potencial conflito de interesses referente a este artigo.
13. Martinez JA, Kearney JM, Kafatos A, Paquet S, Matinez-Gonzales MA. Variables independently associated with self-reported obesity in the European Union. Public Health Nutr 1999; 2: 125-33.

14. Maranhão Neto GA, Polito MD, Lira VA. Fidedignidade entre peso e estatura reportados e medidos e a influência do histórico de atividade física em indivíduos que procuram a prática supervisionada de exercícios. Rev Bras Med Esporte 2005; 11: 141-5.

15. Schimidt MI, Duncan BB, Tavares M, Polanczyk CA, Pellanda L, Zimmer MP. Validity of self-report weight: a study of urban Brazilian adults. Rev Saúde Pública 1993; 27: 271-6.

16. Silveira EA, Araújo CL, Gigante DP, Barros AJD, Lima MS. Validação do peso e altura referidos para o diagnóstico do estado nutricional em uma população de adultos no Sul do Brasil. Cad Saúde Pública 2005; 21: 235-45

17. Dey DK, Rothberg E, Sundh V, Bosaeus I, Steen B. Height and body weight in the elderly. A 25 year longitudinal study of a population aged 70 to 95 years. Eur J Clin Nutr 1999; 53: 905-14.

18. Vailas LI, Nitzke SA, Self-reported versus measured weight and height in a older adult meal program population. J Gerontol A Biol Sci 1998; 53: 481-3.

19. Thomas JR, Nelson JK. Métodos de pesquisa em atividade física. Porto alegre: Artmed, 2002.

20. Alvarez BR, Pavan AL. Alturas e Comprimentos. In: Petroski EL, editor. Antropometria: técnicas e padronizações. Palotti, Porto Alegre, 2003: 31-45.

21. WHO, Word Heath Organization. Obesity status: preventing and managing the global epidemic. Report of a WHO consultation on obesity. Geneva, 1998.

22. Fletcher RH, Fletcher SW, Wagner EH. Epidemiologia Clínica: elementos essenciais. 3 a ed. Artes médicas: Porto Alegre, 1986.

23. Bland JM, Altman DG. Statistical methods for assessing agreement between two methods of clinical measurements. The Lancet 1986; 12: 307-10.

24. Chor D, Coutinho Es, Laurenti R. Reliability of self-reported weight and height among State bank employees. Rev Saúde Pública 1999; 33: 16-23. 